Review

\title{
Recent Advancement on the Optical Properties of Two-Dimensional Molybdenum Disulfide $\left(\mathrm{MoS}_{2}\right)$ Thin Films
}

\author{
Mingxiao Ye, Dustin Winslow, Dongyan Zhang, Ravindra Pandey and Yoke Khin Yap * \\ Department of Physics, Michigan Technological University, 1400 Townsend Drive, Houghton, \\ MI 49931, USA; E-Mails: mye1@ mtu.edu (M.Y.); dwinslow@mtu.edu (D.W.); dozhang@ @mtu.edu (D.Z.); \\ pandey@mtu.edu (R.P.)
}

* Author to whom correspondence should be addressed; E-Mail: ykyap@ mtu.edu; Tel.: +1-906-487-2900; Fax: +1-906-487-2933.

Received: 20 January 2015 / Accepted: 4 March 2015 / Published: 16 March 2015

\begin{abstract}
The emergence of two-dimensional (2D) materials has led to tremendous interest in the study of graphene and a series of mono- and few-layered transition metal dichalcogenides (TMDCs). Among these TMDCs, the study of molybdenum disulfide $\left(\mathrm{MoS}_{2}\right)$ has gained increasing attention due to its promising optical, electronic, and optoelectronic properties. Of particular interest is the indirect to direct band-gap transition from bulk and few-layered structures to mono-layered $\mathrm{MoS}_{2}$, respectively. In this review, the study of these properties is summarized. The use of Raman and Photoluminescence (PL) spectroscopy of $\mathrm{MoS}_{2}$ has become a reliable technique for differentiating the number of molecular layers in $2 \mathrm{D} \mathrm{MoS}_{2}$.
\end{abstract}

Keywords: molybdenum disulfide; electronic band structure; direct band-gap;

Raman spectroscopy; resonant Raman scattering; photoluminescence (PL) spectroscopy

\section{Introduction}

Many bulk crystals exhibit layered atomic structure with strong intra-layer bonds, but with weak van der Waals interaction between layers. Graphite is a representative example in which mechanical exfoliation of mono graphite layers has led to the discovery of graphene [1,2]. These exfoliated, thin layered materials are now categorized as two-dimensional (2D) materials. In addition to graphite, other layered materials have gained significant research attention, including hexagonal boron nitride $(h$-BN), and the transition metal dichalcogenides (TMDCs) such as $\mathrm{MoS}_{2}, \mathrm{MoSe}_{2}, \mathrm{WS}_{2}, \mathrm{WSe} 2$, and their 2D oxides [3]. 
The popularity of $2 \mathrm{D}$ materials is due to their unique electronic and optical properties, which are significantly different from the bulk precursors. For example, pristine graphene is a zero band-gap material [4] with an exceptionally high carrier mobility that exceeds $10^{6} \cdot \mathrm{cm}^{2} \cdot \mathrm{V}^{-1} \cdot \mathrm{S}^{-1}$ at $2 \mathrm{~K}$ [5]. For this reason attempts have been made to use graphene to develop a new ultrathin electrical conductor [6]. Although graphene nanoribbons (GNRs) exhibit a small band-gap due to edge effects, field-effect transistors (FETs) and optoelectronic devices based on GNRs still suffer from a relatively low current on/off ratio (just exceeding $10^{4}$ ) [7]. Although structurally similar to graphene thin layers of $h$-BN (boron nitride nanosheets, BNNSs [8,9]) and their nanotubular structures (boron nitride nanotubes, BNNTs [10-13]) have a $6 \mathrm{eV}$ band-gap, and are therefore not interesting as a material for FETs. In contrast, TMDCs have band-gaps of approximately $1-2 \mathrm{eV}[14,15]$. Furthermore, FETs made from molybdenum disulfide $\left(\mathrm{MoS}_{2}\right)$ have a field-effect mobility of at least $200 \mathrm{~cm}^{2} \cdot \mathrm{V}^{-1} \cdot \mathrm{S}^{-1}$ [16], and high on/off switch ratios of up to $10^{10}$ at room temperature [17]. Although bulk TMDCs have been studied for decades $[14,15,18]$, atomically thick 2D layers of TMDCs have just started to gain attention in the past three years, as indicated by a significant increase in the number of published articles as summarized in Figure 1.

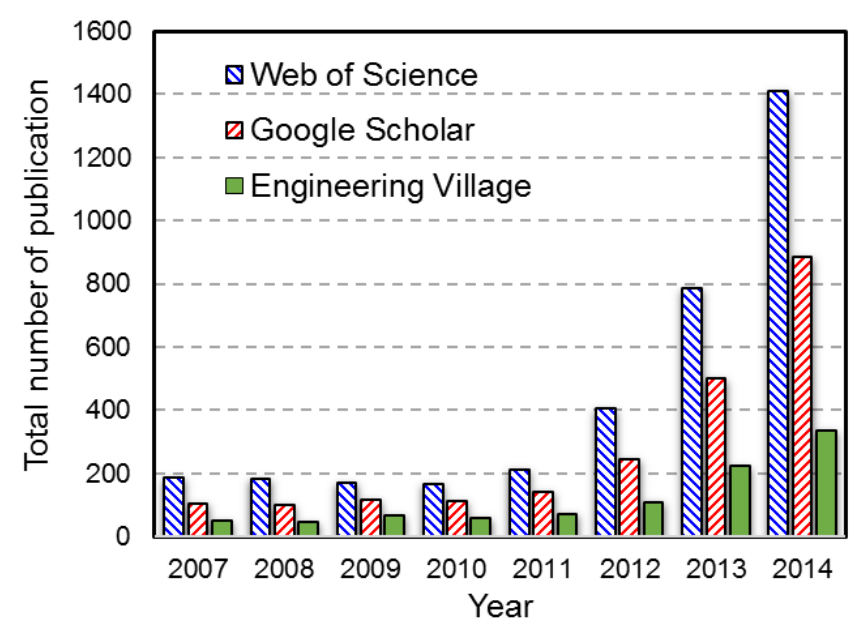

Figure 1. The number of papers published with a title containing or a topic pertaining to molybdenum disulfide $\left(\mathrm{MoS}_{2}\right)$.

Due to the interesting band gap modulation, and a large direct band gap at the visible range, $\mathrm{MoS}_{2}$ is a prospective 2D material for photovoltaic and optoelectronic applications. Therefore, this article will focus on reviewing the optical properties of $\mathrm{MoS}_{2}$ as a representative TMDC. In Section 2, the crystal structure of $\mathrm{MoS}_{2}$ will be described. This is followed by a discussion of the electronic band structure of $\mathrm{MoS}_{2}$, which will prepare readers for the associated optical properties to be discussed in Section 4 (Raman scattering and IR absorption) and Section 5 (resonance Raman scattering).

\section{Crystal Structure}

Molybdenum Disulfide $\left(\mathrm{MoS}_{2}\right)$ belongs to a class of TMDC materials with a formula $\mathrm{MX}_{2}$, where $\mathrm{M}$ is a transition metal element of group IV (Ti, Zr, Hf), group V (V, Nb, Ta), or group VI (Mo, W), and X is a chalcogen $(\mathrm{S}, \mathrm{Se}, \mathrm{Te})$. These materials form layered structures of the form $\mathrm{X}-\mathrm{M}-\mathrm{X}$, with the chalcogen atoms in two hexagonal planes separated by a plane of transition metal atoms. For example, 
$\mathrm{MoS}_{2}$ has a layered structure of covalently bonded S-Mo-S, which is bonded to adjacent layers by weak van der Waals (vdW) interactions between neighboring S-S layers. As shown in Figure 2a, the S and Mo atom layers are hexagonal in structure and each Mo atom is located at the center of a trigonal prism created by six S atoms [19]. Such a tri-layer sandwich structure is considered as a monolayer of $\mathrm{MoS}_{2}$, and is stacked in three possible configurations, with weak vdW S-S bonds as shown in Figure $2 b$, to form multilayer $\mathrm{MoS}_{2}$.

(a)

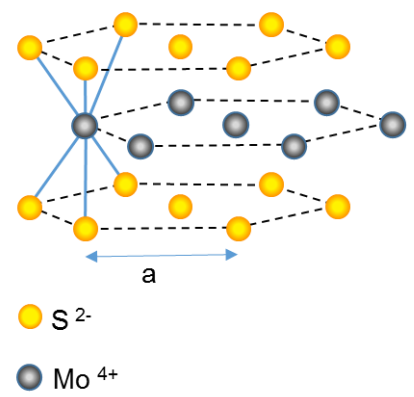

(b)

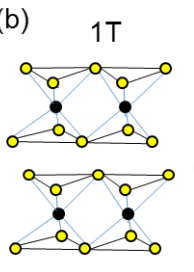

$\circ \mathrm{s}$

- Mo
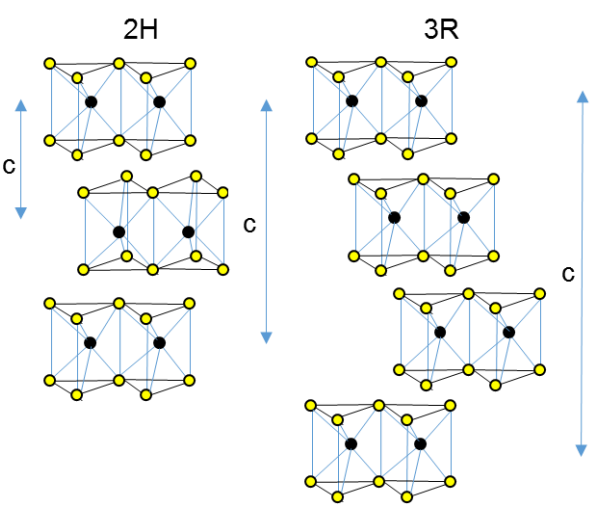

Figure 2. (a) Hexagonal structure of S and Mo layers; (b) Side view schematic illustration of the 1T/2H/3R type structures of $\mathrm{MoS}_{2}$; (c) indicates the number of layers in a repeat unit.

These vdW interactions allow $\mathrm{MoS}_{2}$ to form a bulk crystal of different polytypes, which vary by stacking order and atom coordination. As shown in Figure 2b, there are three known common $\mathrm{MoS}_{2}$ structures. The $2 \mathrm{H}$ and $3 \mathrm{R}$ structures both occur in nature, and have trigonal prismatic coordination [20]. The 3R type has rhombohedra symmetry with three S-Mo-S units. The $2 \mathrm{H}-\mathrm{MoS}_{2}$ has hexagonal symmetry with two S-Mo-S units per primitive cell. The 1T type is a metastable structure discovered in the 1990s [21,22]. It has octahedral coordination with tetragonal symmetry. It has only one $\mathrm{S}-\mathrm{Mo}-\mathrm{S}$ as a repeat cell. Both the $1 \mathrm{~T}$ and $3 \mathrm{R}$ types are metastable, and they can change to the $2 \mathrm{H}-\mathrm{MoS}_{2}$ structure through heating $[23,24]$. All of the bulk $\mathrm{MoS}_{2}$ crystals discussed hereafter are of the $2 \mathrm{H}-\mathrm{MoS}_{2}$ structure.

\section{Electronic Band Structure: Optical and Optoelectronic Properties}

The electronic characteristics of TMDCs range from metal to semiconductor [14,15,25-27]. Many TMDCs' electronic band structures have been theoretically investigated through the use of several modeling techniques. In the 1970s, semi-empirical calculations based on the tight-binding method were made by Bromley, Murray, and Yoffe without taking into account interlayer interaction effects [28,29]. From the 1970s to the present, $a b$ initio and first principles calculations using density functional theory (DFT) with different approximations (e.g., local density approximations (LDA)) were made and widely used [30-40]. These models were guided by a variety of spectroscopic tools [14,18,24,27,41-50]. 


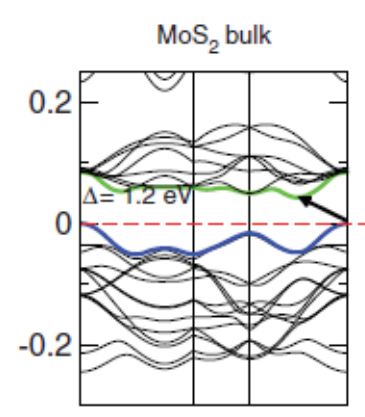

$\mathrm{MoS}_{2}$ quadrilayer

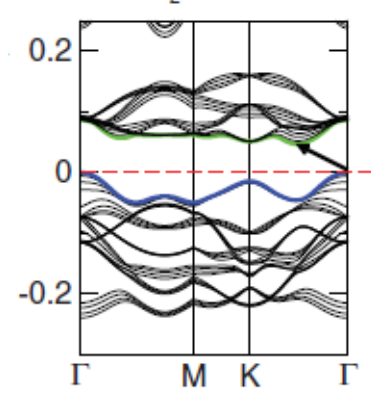

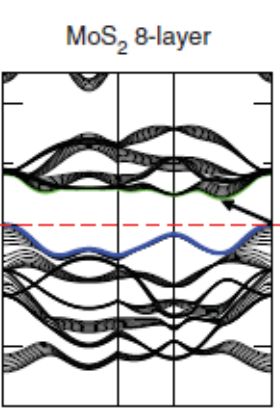

$\mathrm{MoS}_{2}$ bilayer

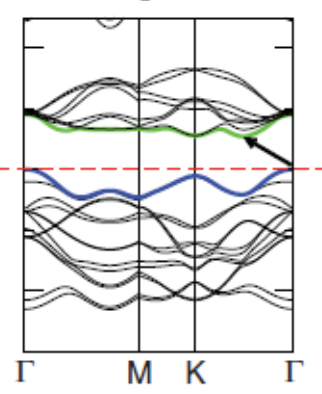

$\mathrm{MoS}_{2}$ 6-layer

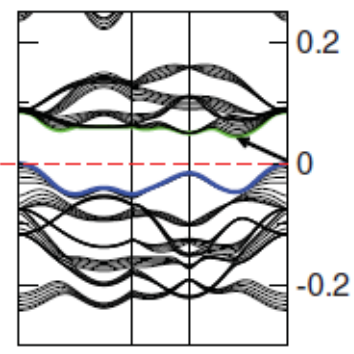

$\mathrm{MoS}_{2}$ monolayer

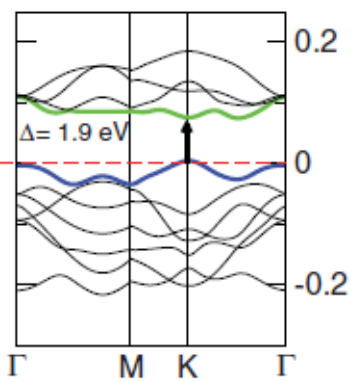

Figure 3. Band structure of different layers of $\mathrm{MoS}_{2}$ [36]. Reprinted figure with permission from [Kuc, A.; Zibouche, N.; et al. Influence of quantum confinement on the electronic structure of the transition metal sulfide $\mathrm{TS}_{2}$. Phys. Rev. B 2011, 83, 245213] Copyright (2011) by the American Physical Society.

Recently, $\mathrm{MoS}_{2}$ has been of particular interest. As shown in Figure 3, bulk $\mathrm{MoS}_{2}$ is an indirect band gap semiconductor with a band gap of $1.2 \mathrm{eV}$, which originates from the top of the valence band situated at the $\Gamma$ point to the bottom of the conduction band halfway between the $\Gamma$ and $\mathrm{K}$ points. It should be noted that there is a direct band gap situated at the bulk $\mathrm{MoS}_{2} \mathrm{~K}$ point. As the number of layers decreases, the fundamental indirect band-gap (from the $\Gamma$ point to halfway between the $\Gamma$ point and the $\mathrm{K}$ point) increases due to the quantum confinement effect. In the case of monolayer $\mathrm{MoS}_{2}$, it becomes larger than the direct band-gap located at the K-point, which has been changed by less than $0.1 \mathrm{eV}$ as the number of layers was reduced. In the monolayer limit, $\mathrm{MoS}_{2}$ changes from a bulk indirect band-gap semiconductor to a $2 \mathrm{D}$ direct band-gap semiconductor. The indirect band-gap of bulk $\mathrm{MoS}_{2}(1.2 \mathrm{eV})$ is supplanted by the direct band-gap $(1.9 \mathrm{eV})$ at the monolayer $\mathrm{MoS}_{2} \mathrm{~K}$-point, as shown in Figure 3.

This layer number-dependent band-structure is due to quantum confinement in the c-axis of the crystal. The band-gap change with film thickness was predicted by Sandomirskii in 1963; the band-gap of semiconducting films was shown to change by $\Delta E_{g}=\frac{\hbar^{2} \pi^{2}}{2 m a^{2}}$, where a is the film thickness [51,52]. Quantum confinement of carriers was also studied in ultrathin film $\mathrm{MoS}_{2}$ and other TMDCs [53-55]. The out-of-plane effective mass for electrons and holes around the $\mathrm{K}$ point is far exceeded by the free electron and hole mass $\left(\mathrm{m}_{0}\right)$. Whereas the hole effective mass around the $\Gamma$ point is estimated to be $0.4 \mathrm{~m}_{0}$, and the electron effective mass of the conduction band minimum along the $\Gamma-\mathrm{K}$ direction is only $0.6 \mathrm{~m}_{0}$ [46]. Therefore, the decrease of thickness to monolayer leads to significant quantum confinement. To explain this result, Mak et al. applied a zone-folding scheme to describe the electronic band structure of ultrathin $\mathrm{MoS}_{2}$ film [46]. As shown in Figure 4a, the allowed electronic state's wave vector (out-of-plane 
momentum k) for monolayer $\mathrm{MoS}_{2}$ can directly occur in the A-H direction. By increasing the $\mathrm{MoS}_{2}$ thickness, the out-of-plane momentum $\mathrm{k}$ will approach the $\Gamma$-K direction (Figure $4 \mathrm{~b}$ ). In this case the top of the conduction band and the bottom of the valence band occur at the $\Gamma$ point and along the $\Gamma-\mathrm{K}$ direction. The d orbitals' contribution to the band structure of $\mathrm{MoS}_{2}$ has been of particular research interest $[25,28,32,33,42,56]$. The $2 \mathrm{H}-\mathrm{MoS}_{2}$ with trigonal prismatic coordination has a filled $d_{z^{2}}$ valence band that overlaps the filled sp orbitals of the $S$ atoms. The conduction band is derived from the degenerate $d_{x^{2}-y^{2}}$ and $d_{x}$ orbitals that overlap the empty anti-bonding sp orbitals of the $\mathrm{S}$ atoms. Based on density of state (DOS) data [34-37,39], the valence and conduction bands near the $\Gamma$ point were found to be a linear combination of $d$ orbitals of the Mo atoms and $\mathrm{p}_{z}$ orbitals of the $\mathrm{S}$ atoms. At the $\mathrm{K}$ point, the valence and conduction band states are primarily composed of the $\mathrm{d}$ orbitals of the Mo atoms, which are located in the middle of the S-Mo-S unit. Compared to the Mo atoms, the S atoms have strong interlayer coupling; their electron state energy depends on layer thickness, and leads to a direct band-gap structure at the $\mathrm{K}$ point when the layer thickness decreases.

(a)

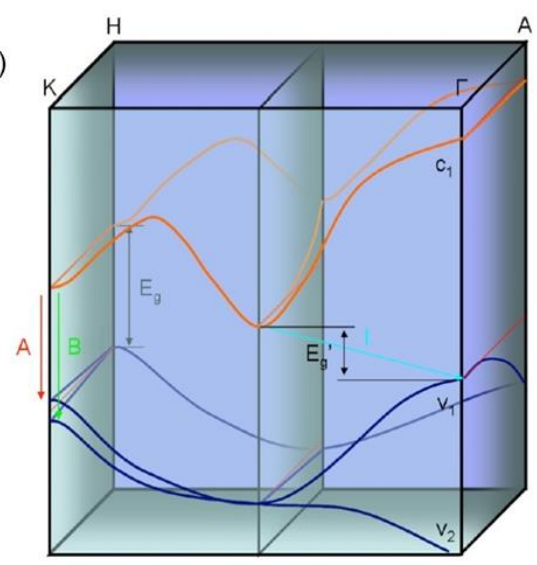

(b)

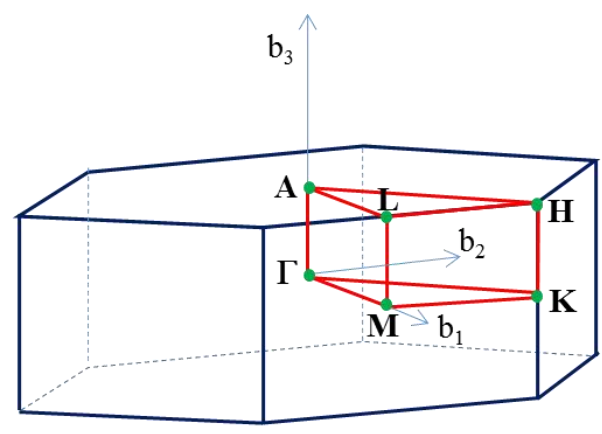

Figure 4. (a) Simplified electronic band structure of bulk $\mathrm{MoS}_{2}$ [46]. Reprinted figure with permission from [Mak, K.F.; Lee, C.; James, H.; Jie, S.; Heinz, T.F. Atomically Thin $\mathrm{MoS}_{2}$ : A New Direct-Gap Semiconductor. Phys. Rev. Lett. 2010, Phys. Rev. Lett. 105.136805] Copyright (2010) by the American Physical Society. (b) Brillouin zone and special point of the hexagonal lattice system $\left(\mathrm{MoS}_{2}\right)$ [57].

This and other layer-dependent band structure variation in TMDCs has recently seen increased research interest, particularly their optical and optoelectronic properties. For instance, as a result of the direct band-gap, photons with energy greater than the band-gap can be easily absorbed. Hence there is an obvious change in the photoluminescence, absorption, and photoconductivity spectra for ultrathin film $\mathrm{MoS}_{2}$, especially for monolayer $\mathrm{MoS}_{2}[24,46,47,49]$, as shown in Figure 5. This phenomenon allows the possibility of using $\mathrm{MoS}_{2}$ for optoelectronic applications [58].

As shown in Figure 5a, the photoluminescence peaks between $600 \mathrm{~nm}$ and $700 \mathrm{~nm}$ become stronger with the reduction in the number of layer of $\mathrm{MoS}_{2}$. The corresponding absorption peaks can be identified as shown in Figure 5b. Both of them correspond to A and B exciton transitions in the direct band-gap structure $[14,18,59]$. A clear indirect to direct band structure transition between bilayer and monolayer $\mathrm{MoS}_{2}$ can be best seen from the photocurrent spectra shown in Figure 5c. The direct band-gap energies 
of $E_{A}$ and $E_{B}$, as measured by different methods, are summarized in Table 1. Initially, the research was in agreement that the $\mathrm{A}$ and $\mathrm{B}$ exciton transitions occur at the $\Gamma$ point [60-62]. There was some disagreement from M. R. Khan and G. J. Goldsmith's work in 1983, which put the A and B exciton transition at the A point of the Brillouin Zone [63]. However, further calculations have shown that the A and $\mathrm{B}$ exciton transitions at the $\mathrm{K}$ point $[30,32,64-66]$. The $\mathrm{A}$ and $\mathrm{B}$ exciton energy difference is due to the $d-d$ transitions from the filled $d_{x}$ orbital to the degenerated $d_{x y}$ and $d_{x}^{2}-y^{2}$ orbitals, which split by spin-orbit coupling [62,67].

(a)

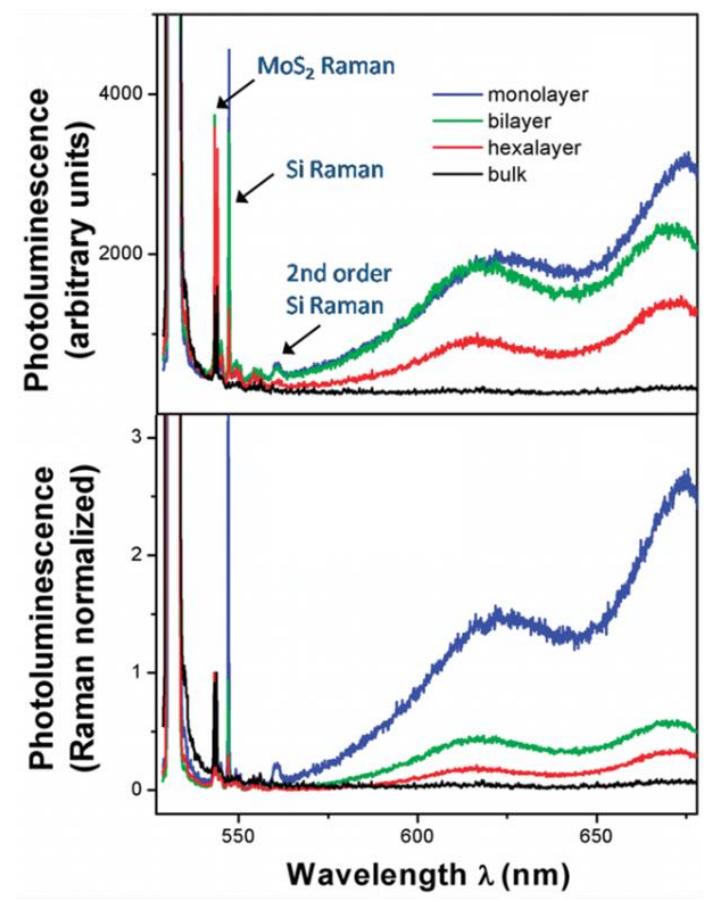

(b)
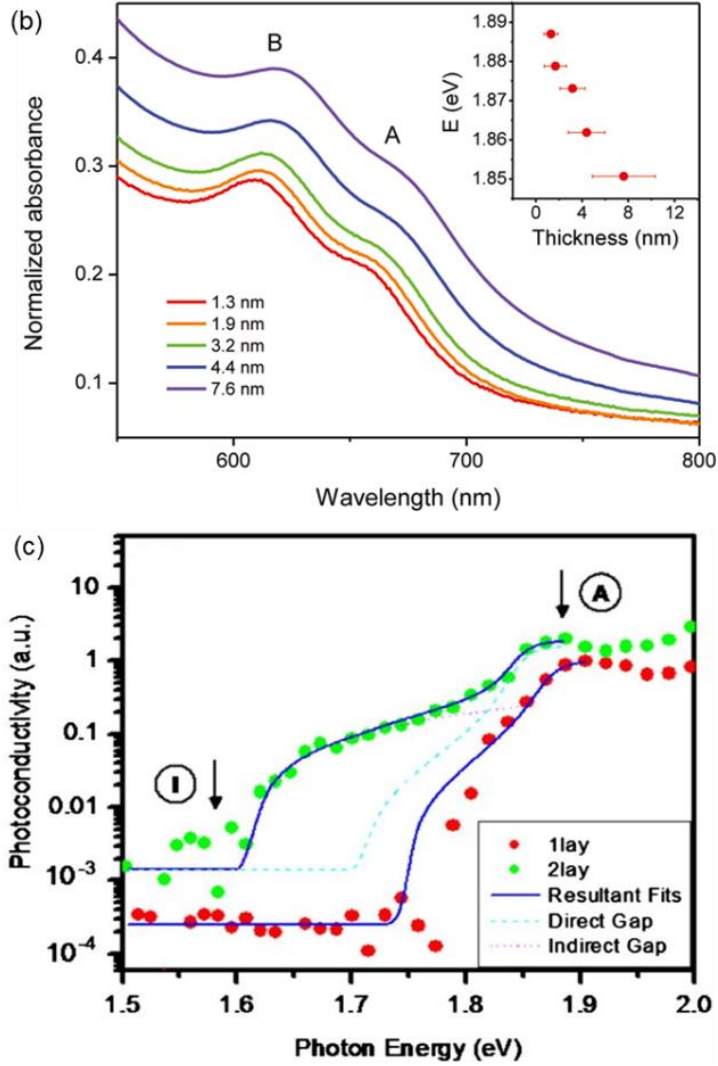

Figure 5. (a) Photoluminescence spectra from monolayer to bulk $\mathrm{MoS}_{2}$ [47]. Reprinted figure with permission from [Splendiani, A.; Sun, L.; Zhang, Y.; Li, T.; Kim, J.; Chim, C.-Y.; Galli, G.; Wang, F. Emerging photoluminescence in monolayer $\mathrm{MoS}_{2}$. Nano Lett. 2010, 10, 1271-1275] Copyright (2010) by the American Chemical Society. (b) Absorption spectra of ultrathin film $\mathrm{MoS}_{2}$ from $1.3 \mathrm{~nm}$ to $7.6 \mathrm{~nm}$. Inset figure shows the A exciton energy with film thickness [24]. Reprinted figure with permission from [Eda, G.; Yamaguchi, H.; Voiry, D.; Fujita, T.; Chen, M.; Chhowalla, M. Photoluminescence from chemically exfoliated $\mathrm{MoS}_{2}$. Nano Lett. 2011, 11, 5111-5116.] Copyright (2011) by the American Chemical Society. (c) Photoconductivity spectra of monolayer and bilayer $\mathrm{MoS}_{2}$ [46]. Reprinted figure with permission from [Mak, K.F.; Lee, C.; James, H.; Jie, S.; Heinz, T.F. Atomically Thin $\mathrm{MoS}_{2}$ : A New Direct-Gap Semiconductor. Phys. Rev. Lett. 2010, Phys. Rev. Lett. 105.136805] Copyright (2010) by the American Physical Society. 
As a $2 \mathrm{D}$ material, monolayer $\mathrm{MoS}_{2}$ is expected to have strong excitonic effects, which will affect the optical properties. One of the commonly used calculation methods is the GW plus Bethe-Salpeter equation (GW-BSE), which computes quasiparticle band structure and optical response, including electron-electron/hole interactions. Although the previously reported experimental and theoretical band gap for $\mathrm{MoS}_{2}$ is around $1.9 \mathrm{eV}$, recent calculations based on the $\mathrm{GW}$ method for monolayer $\mathrm{MoS}_{2}$ have shown that the quasiparticle band-gap is larger. However, the calculated results vary widely between 2.2 and $2.8 \mathrm{eV}$ [37,66,68-72]. Currently there is no experimental proof for the larger quasiparticle band-gap in $\mathrm{MoS}_{2}$, although evidence was recently demonstrated for $\mathrm{WS}_{2}$ [73].

Table 1. There is a wide range of published values of direct band-gap energy $E_{A 1}$ and $E_{B 1}$ measured at different temperatures.

\begin{tabular}{|c|c|c|c|}
\hline Materials / Method & $E_{A 1}(e V)$ & $\mathbf{E}_{B 1}(\mathrm{eV})$ & Temperature (K) \\
\hline \multirow[t]{2}{*}{$2 \mathrm{H}-\mathrm{MoS}_{2} /$ piezo reflectance $[74]$} & $1.929 \pm 0.005$ & $2.136 \pm .008$ & 25 \\
\hline & $1.845 \pm 0.008$ & $2.053 \pm 0.01$ & 300 \\
\hline $2 \mathrm{H}-\mathrm{MoS}_{2} /$ reflectance $[14,75,76]$ & 1.88 & 2.06 & 300 \\
\hline $\begin{array}{c}\text { 2H-MoS } 2 \text { / wavelength modulation } \\
\text { reflectance (WMR) [77] }\end{array}$ & 1.9255 & 2.137 & 4.2 \\
\hline 2H-MoS 2 / photoconductivity [77] & 1.92 & 2.124 & 4.2 \\
\hline 2H-MoS 2 / absorption [78] & 1.9449 & 2.1376 & 7 \\
\hline 2H-MoS 2 / absorption [44] & 1.90 & 2.10 & 150 \\
\hline 2H-MoS 2 / absorption [44] & 1.91 & 2.11 & $75 / 25$ \\
\hline $\begin{array}{c}\text { Inorganic Fullerene like } 2 \mathrm{H}-\mathrm{MoS}_{2} \\
\text { absorption [79] }\end{array}$ & 1.82 & 1.95 & Room \\
\hline $\begin{array}{c}\text { 2H-MoS } 2 \text { / Bethe-Slapeter Equation (BSE) } \\
\text { Calculation [66] }\end{array}$ & 1.78 & 1.96 & - \\
\hline Monolayer $\mathrm{MoS}_{2}$ / absorption [46] & 1.88 & 2.03 & Room \\
\hline Ultrathin-MoS 2 / photoluminescence [47] & 1.85 & 1.98 & Room \\
\hline
\end{tabular}

\section{Lattice Dynamic: Raman Scattering and IR Absorption}

Next we consider the lattice dynamics (Figure 6) in $\mathrm{MoS}_{2}$ that correspond to the phonon modes that contribute to Raman scattering and IR absorption. As was illustrated in Figure 2, $\mathrm{MoS}_{2}$ consists of two hexagonal planes of $\mathrm{S}$ atoms and an intercalated hexagonal plane of Mo atoms. The symmetry point group of the $S$ atoms is $C_{3 v}$, while the symmetry of the Mo atoms is point group $D_{3 h}$. Furthermore, the irreducible representations of $\mathrm{C}_{3 \mathrm{v}}$ and $\mathrm{D}_{3 \mathrm{~h}}$ are related to factor group $\mathrm{D}_{6} \mathrm{~h}$ [80]. Therefore, MoS 2 with even numbers of layers or bulk crystal have a symmetry similar to the $\mathrm{D}_{6} \mathrm{~h}$ point group, and systems with odd numbers of layers (including monolayer crystals) have D3h space group symmetry (without inversion symmetry) [38]. 


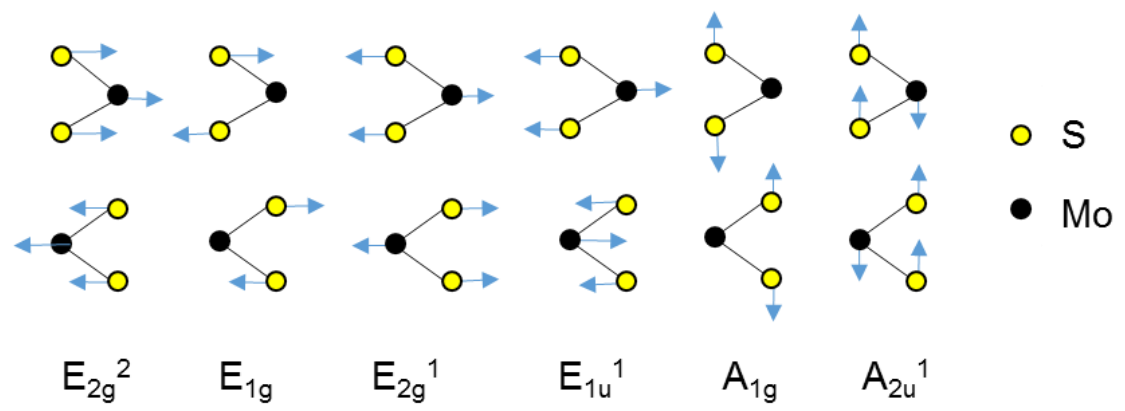

Figure 6. Atomic displacement vector of the four first-order Raman-active $E_{2 g}{ }^{2}, E_{1 g}, E_{2 g}{ }^{1}, A_{1 g}$ modes and two IR-active $\mathrm{E}_{1 \mathrm{u}}{ }^{1}$ and $\mathrm{A}_{2 \mathrm{u}}{ }^{1}$ modes, as viewed along the [100] direction [81-83].

As shown in Table 2, there are four first-order Raman active modes that are present in most reported $\mathrm{MoS}_{2}$ Raman spectroscopy studies, $\mathrm{A}_{1 \mathrm{~g}}, \mathrm{E}_{2 \mathrm{~g}}{ }^{1}, \mathrm{E}_{2 \mathrm{~g}}{ }^{2}$, and $\mathrm{E}_{1 \mathrm{~g}}$. As shown in Figure 6, the $\mathrm{A}_{1 \mathrm{~g}}$ mode is due to the out-of-plane vibration among the $\mathrm{S}$ atoms as viewed along the [100] direction. The other three Raman active modes are due to in-plane vibrations, illustrated in Figure 6. Among the four Raman-active modes the $\mathrm{A}_{1 \mathrm{~g}}$ and $\mathrm{E}_{2 \mathrm{~g}}{ }^{1}$ modes near $400 \mathrm{~cm}^{-1}$ are readily observable. The $\mathrm{E}_{1 \mathrm{~g}}$ mode and $\mathrm{E}_{2 \mathrm{~g}}{ }^{2}$ mode usually cannot be detected by conventional Raman Scattering measurements.

Table 2. Relevant phonon irreducible symmetry representations of $S$ atoms $\left(C_{3 v}\right)$, Mo atoms $\left(\mathrm{D}_{3 \mathrm{~h}}\right)$, and bulk $2 \mathrm{H}-\mathrm{MoS}_{2}$ point group $\mathrm{D}_{6 \mathrm{~h}}$. This table is compiled from the work of T. J. Weiting et al. [80], A.M. Sanchez et al. [38], and T. Livneh et al. [84]. Some vibration frequency data were measured by $[85,86]$.

\begin{tabular}{|c|c|c|c|c|c|c|c|}
\hline $\mathrm{C}_{3 \mathrm{v}}(\mathrm{S})$ & $\mathbf{D}_{3 \mathbf{h}}(\mathbf{M o})$ & $\mathrm{D}_{6 \mathrm{~h}}\left(\mathrm{MoS}_{2}(\Gamma)\right)$ & $\begin{array}{c}\text { Transformation } \\
\text { Properties }\end{array}$ & Activity & $\begin{array}{l}\text { Vibration } \\
\text { Direction }\end{array}$ & $\begin{array}{c}\text { Atoms } \\
\text { Involved }\end{array}$ & Frequency $\left(\mathrm{cm}^{-1}\right)$ \\
\hline \multirow[t]{2}{*}{$\mathrm{A}_{1}$} & $A_{1}$, & $A_{1 g}$ & $\left(\alpha_{x x}+\alpha_{y y}, \alpha_{z z}\right)$ & Raman & Out & $S$ & 409 \\
\hline & $\mathrm{A}_{2}$ & $\mathrm{~B}_{1 \mathrm{u}}$ & - & Inactive & Out & $S$ & 403 \\
\hline \multirow[t]{4}{*}{$\mathrm{A}_{2}$} & $\mathrm{~A}_{1}{ }^{\prime}$ & $\mathrm{B}_{2 \mathrm{~g}}{ }^{1}$ & - & Inactive & Out & $\mathrm{Mo}+\mathrm{S}$ & 475 \\
\hline & & $\mathrm{B}_{2 \mathrm{~g}}{ }^{2}$ & - & Inactive & Out & $\mathrm{Mo}+\mathrm{S}$ & 56 \\
\hline & $\mathrm{A}_{2}{ }^{\prime}$ & $\mathrm{A}_{2 \mathrm{u}}{ }^{1}$ & $\mathrm{~T}_{\mathrm{z}}$ & $\operatorname{IR}(E \| c)$ & Out & $\mathrm{Mo}+\mathrm{S}$ & 470 \\
\hline & & $\mathrm{A}_{2 \mathrm{u}} \mathrm{u}^{2}$ & $\mathrm{~T}_{\mathrm{z}}$ & Acoustic & Out & $\mathrm{Mo}+\mathrm{S}$ & - \\
\hline \multirow[t]{6}{*}{$\mathrm{E}$} & $E^{\prime}$ & $\mathrm{E}_{2 \mathrm{~g}}{ }^{1}$ & $\left(\alpha_{x x}-\alpha_{y y}, \alpha_{z z}\right)$ & Raman & In & $\mathrm{Mo}+\mathrm{S}$ & 383 \\
\hline & & $\mathrm{E}_{1 \mathrm{u}}{ }^{1}$ & $\left(\mathrm{~T}_{\mathrm{x}}, \mathrm{T}_{\mathrm{y}}\right)$ & $\mathrm{IR}(\mathrm{E} \perp \mathrm{c})$ & In & $\mathrm{Mo}+\mathrm{S}$ & 384 \\
\hline & & $\mathrm{E}_{2 \mathrm{~g}}{ }^{2}$ & $\left(\alpha_{x x}-\alpha_{y y}, \alpha_{z z}\right)$ & Raman & In & $\mathrm{Mo}+\mathrm{S}$ & 34 \\
\hline & & $\mathrm{E}_{1 \mathrm{u}}^{2}$ & $\left(\mathrm{~T}_{\mathrm{x}}, \mathrm{T}_{\mathrm{y}}\right)$ & Acoustic & In & $\mathrm{Mo}+\mathrm{S}$ & - \\
\hline & E', & $E_{1 g}$ & $\left(\alpha_{y z}, \alpha_{z x}\right)$ & Raman & In & $S$ & 286 \\
\hline & & $E_{2 u}$ & - & Inactive & In & $S$ & 297 \\
\hline
\end{tabular}

The weak intensity of the $E_{1 \mathrm{~g}}$ mode is due to the laser polarization chosen and random crystal direction of different $\mathrm{MoS}_{2}$ samples [86]. For example, the $\mathrm{E}_{1 \mathrm{~g}}$ mode is forbidden for s-polarized laser light incident on the $\mathrm{MoS}_{2}$ crystal surface (the E field is perpendicular to the c-axis of $\mathrm{MoS}_{2}$ ) [85]. In 1970, J. L. Verbel and T. J. Wieting studied the $E_{1 \mathrm{~g}}$ mode, and predicted that it would be located around $519 \mathrm{~cm}^{-1}$ [83]. However, after correcting for the polarization effect in 1971 they discovered the very weak line at $287 \mathrm{~cm}^{-1}$ [80]. This was confirmed by J. M. Chen and C. S. Wang using back scattering 
geometry [87,88], which placed the $\mathrm{E}_{1 \mathrm{~g}}$ mode at $286 \mathrm{~cm}^{-1}$. This $\mathrm{E}_{\mathrm{g}}$ mode has been shown to be more intense than the other three first-order Raman active modes, which are activated with the use of a p-polarized laser. Conversely, the $\mathrm{E}_{1 \mathrm{~g}}$ mode intensity is much weaker with the use of an s-polarized laser [85].

As shown in Figure 6, the $\mathrm{E}_{2 \mathrm{~g}}{ }^{2}$ mode is due to the vibrational interaction of adjacent rigid layers within the $\mathrm{MoS}_{2}$ crystal. For this reason it is difficult to observe the $\mathrm{E}_{2 \mathrm{~g}}{ }^{2}$ peak at low wavenumber, because of the presence of strong Rayleigh scattering. The corresponding Raman scattering peak for the $\mathrm{E}_{2 \mathrm{~g}^{2}}$ mode is located at $33.7( \pm 1) \mathrm{cm}^{-1}$ and was first experimentally observed by J. L. Verbel, T. J. Wieting, and P. R. Reed by use of a triple Raman spectrometer [89].

In addition to the four first-order Raman active modes $\left(\mathrm{A}_{1 \mathrm{~g}}, \mathrm{E}_{2 \mathrm{~g}}{ }^{1}, \mathrm{E}_{2 \mathrm{~g}}{ }^{2}\right.$, and $\mathrm{E}_{1 \mathrm{~g}}$ ) there are four degeneracy lattice modes- $\mathrm{E}_{2 \mathrm{u}}, \mathrm{E}_{1 \mathrm{u}}{ }^{1}, \mathrm{~B}_{1 \mathrm{u}}$, and $\mathrm{B}_{2 \mathrm{~g}}{ }^{2}$ - that are Raman inactive. Among these, $\mathrm{E}_{1 \mathrm{u}}{ }^{1}$ and $\mathrm{A}_{2 \mathrm{u}}{ }^{1}$ are IR active modes [83].
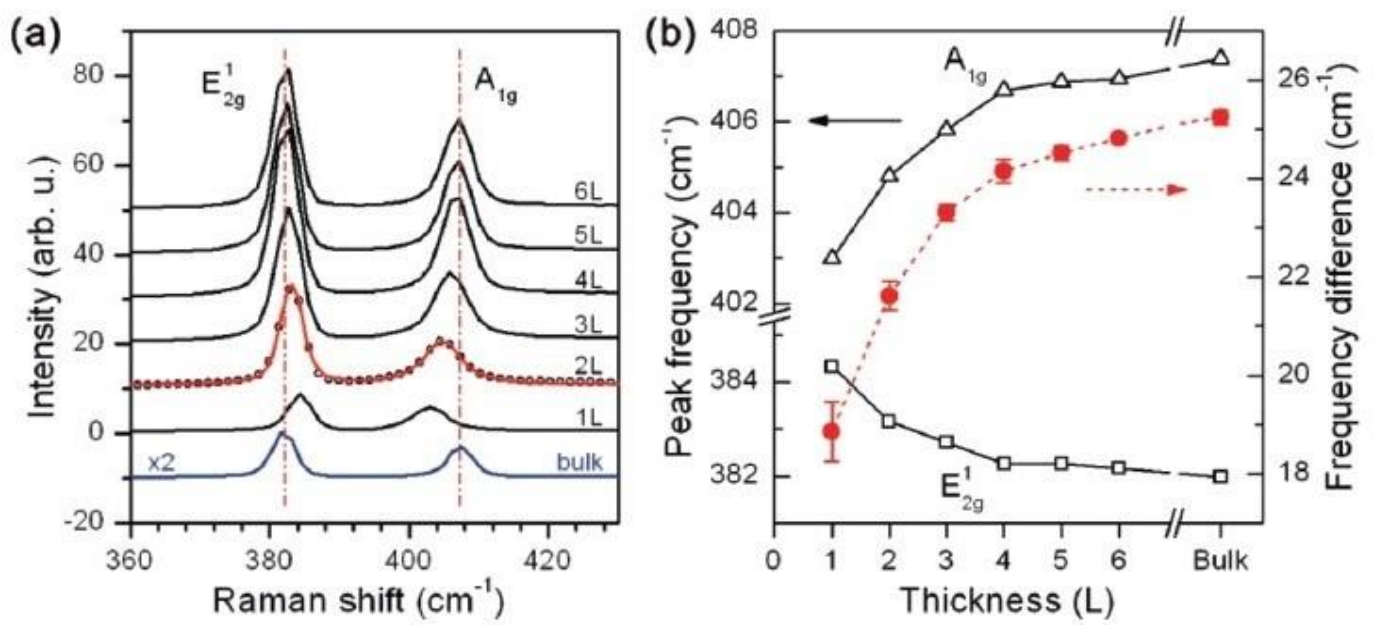

Figure 7. (a) Raman spectrum for different thicknesses of $\mathrm{MoS}_{2}$. (b) Frequencies of $\mathrm{A}_{1 \mathrm{~g}}$ and $\mathrm{E}_{2 \mathrm{~g}}{ }^{1}$ modes against layer thickness [82]. Reprinted figure with permission from [Lee, C.; Yan, H.; Brus, L.E.; Heinz, T.F.; Hone, J.; Ryu, S. Anomalous lattice vibrations of singleand few-layer MoS2. ACS Nano 2010, 4, 2695-2700] Copyright (2010) by the American Chemical Society.

It should be noted that most Raman spectra show strong signals for both the $\mathrm{E}_{2 \mathrm{~g}}{ }^{1}$ and $\mathrm{A}_{1 \mathrm{~g}}$ modes, as shown in Figure 7a. To explain the vibrational modes of $\mathrm{E}_{2 \mathrm{~g}}{ }^{1}$ and $\mathrm{A}_{1 \mathrm{~g}}$, many classical models have been introduced. For example, Bromley used a Born-Von model in the nearest neighbor approximation in an attempt to explain this observation [90]. On the other hand, T. J. Wieting and J. L. Verble found that the classical dielectric oscillator model fits well with the infrared reflectivity and Raman scattering data [80]. This model fits well because the interlayer force is about 100 times smaller than the intralayer force [89], and therefore one only needs to consider the interatomic forces within a single layer. Furthermore, because of the small effective charge, the coulomb force is negligible [80]. However, C. Lee et al. found that these two modes exhibited abnormal film thickness dependence, as shown in Figure 7 [81]. For instance, as shown in Figure $7 \mathrm{~b}$, when the film thickness is increased the frequency of the $\mathrm{E}_{2 \mathrm{~g}}{ }^{1}$ mode decreases (red shifts) while the $\mathrm{A}_{1 \mathrm{~g}}$ increases (blue shifts). This width-dependent frequency shift means 
that the $\mathrm{E}_{2 \mathrm{~g}}{ }^{1}$ and $\mathrm{A}_{1 \mathrm{~g}}$ frequency are better for identifying ultrathin $\mathrm{MoS}_{2}$ thickness than either peak intensity or width for no more than four layers [91]. This result is consistent with optical microscope, atomic force microscopy (AFM), and photoluminescence measurements [47,81,91]. Based on various published sources, we have summarized in Table 3 the $\mathrm{E}_{2 \mathrm{~g}}{ }^{1}$ and $\mathrm{A}_{\mathrm{gg}}$ mode frequencies detected from bulk and thin layers of $\mathrm{MoS}_{2}$ by various laser wavelengths.

The dependence of the $\mathrm{E}_{2 \mathrm{~g}}{ }^{1}$ and $\mathrm{A}_{1 \mathrm{~g}}$ modes' frequency on the layer thickness of $\mathrm{MoS}_{2}$ can be explained using the van der Waals force model. As the number of layers is increased, the restoring force between inter-layer S-S bonds will enhance, which fits the observed trend of the out-of-plane A1g mode frequency increasing. However, the $\mathrm{E}_{2 \mathrm{~g}}{ }^{1}$ peak shows a red-shift as the number of layers is increased, which suggests that the classical model for coupled harmonic oscillators is inadequate and there is an additional interlayer interaction. One reason for the inadequacy of the classical model is that it only considers weak interlayer interaction. However, the properties of the $\mathrm{MoS}_{2}$ layers might be different from those on the surface of bulk $\mathrm{MoS}_{2}$. This is consistent with surface phenomenon measurements investigated by P. A. Bertrand, who reported the surface phonon dispersion of $\mathrm{MoS}_{2}$ as measured by high resolution electron-energy-loss spectroscopy (HREELS). It was found that the peak energy of the Alg optical mode for thin layers is 3.1 $( \pm 0.2)$ meV lower than the related bulk phonon [92].

Table 3. Summary of the $\mathrm{E}_{2 \mathrm{~g}}{ }^{1}$ and $\mathrm{A}_{1 \mathrm{~g}}$ mode frequency of $\mathrm{MoS}_{2}$ as excited by different laser sources and varied by the number of layers [91]. The $514.5^{\#} \mathrm{~nm}$ data is extracted from C. Lee et al. [82]. The $514.5 \mathrm{~nm}^{*}$ and $632.8 \mathrm{~nm}^{*}$ data only have 1/2/4/7/bulk layers [93].

\begin{tabular}{|c|c|c|c|c|c|c|c|c|c|c|}
\hline \multirow{2}{*}{$\begin{array}{c}\text { Laser } \\
(\mathrm{nm})\end{array}$} & \multicolumn{5}{|c|}{$\mathbf{E}_{2 \mathrm{~g}}{ }^{1}$ Mode Frequency $\left(\mathrm{cm}^{-1}\right)$} & \multicolumn{5}{|c|}{ A1g Mode Frequency $\left(\mathrm{cm}^{-1}\right)$} \\
\hline & $1 \mathrm{~L}$ & $2 \mathrm{~L}$ & $3 \mathrm{~L}$ & $4 \mathrm{~L}$ & bulk & $1 \mathrm{~L}$ & $2 \mathrm{~L}$ & $3 \mathrm{~L}$ & $4 \mathrm{~L}$ & bulk \\
\hline 325 & 384.2 & 382.8 & 382.8 & 382.7 & 382.5 & 404.9 & 405.5 & 406.3 & 407 & 407.8 \\
\hline 488 & 384.7 & 383.3 & 383.2 & 382.9 & 383 & 402.8 & 405.5 & 406.5 & 407.4 & 408 \\
\hline $514.5^{\#}$ & 384.3 & 383.2 & 382.7 & 382.3 & 382 & 403 & 404.8 & 405.8 & 406.7 & 407.8 \\
\hline $514.5^{*}$ & 386.1 & 383.1 & - & 383.7 & 383.3 & 404.7 & 406.8 & - & 408 & 408.6 \\
\hline 532 & 384.7 & 382.5 & 382.4 & 382.4 & 383 & 402.7 & 404.9 & 405.7 & 406.7 & 407.8 \\
\hline 632.8 & 385 & 383.8 & 383.3 & 382.9 & 381.5 & 403.8 & 404.8 & 405 & 406 & 406.6 \\
\hline 632.8* & 386.4 & 383.1 & - & 383.3 & 382.8 & 405 & 406.2 & - & 407.3 & 408.3 \\
\hline
\end{tabular}

In fact, the red shift of the $\mathrm{E}_{2 \mathrm{~g}}{ }^{1}$ peak with the increase in layer thickness is due to the fact that thinner $\mathrm{MoS}_{2}$ has a shorter intra-layer S-Mo-S distance. In 1977, B. J. Mrstik et al. found surface reconstruction in $\mathrm{MoS}_{2}$ by the use of low-energy electron diffraction (LEED). These LEED measurements showed that the topmost intra-layer space between S-Mo-S atoms of thin $\mathrm{MoS}_{2}$ contracts up to $5 \%$ compared to that in bulk planes [94]. These results show that layer stacking effects change the intralayer structure. Moreover, A. M. Sanchez and L. Wertz introduced long-range Coulomb interlayer interactions into models of multilayer $\mathrm{MoS}_{2}$ to explain the $\mathrm{E}_{2 \mathrm{~g}}{ }^{1}$ anomalous red-shift when the thickness is increased [38]. It was previously introduced to explain the vibrational mode of $\beta$-GaSe by T. J. Wieting et al. [95]. From these models it is apparent that the interlayer force changes in two ways when moving from monolayer to bulk samples. The short-range term of the force slightly increases because of the enhanced restoring interaction between adjacent layers. The long-range term of the force will decrease, because the layers 
extend over the effective charge, which leads to Coulombic screening [96]. However, this effect mainly happens between the Mo atoms. From Figure 6 the $\mathrm{E}_{2 \mathrm{~g}}{ }^{1}$ mode involves the in-plane vibration of Mo atoms. Consequently, the considerable decrease in the Coulombic, long-range interaction between the Mo atoms overcompensates for the slight increase of the short-range weak interlayer interaction. On the other hand, the $\mathrm{A}_{1 \mathrm{~g}}$ mode is not influenced by the Coulomb potential screening, because the A $1 \mathrm{~g}$ mode only involves out-of-plane vibration of the $\mathrm{S}$ atoms.

\section{Resonant Raman Scattering}

Besides the four first-order Raman modes and their degeneracy modes, J. M. Chen and C. S. Wang have noted that additional second-order modes appear when an Argon $514.5 \mathrm{~nm}$ laser source is used [85]. Furthermore, they found that the $\mathrm{A}_{1 \mathrm{~g}}$ mode combines with a weak longitudinal acoustic phonon mode at the $\mathrm{M}$ point of the Brillouin zone (LA(M)) corroborating inelastic neutron scattering data from Wakabayashi et al. [97].

In 1984, A. M. Stacy and D. T. Hodul presented Raman Spectra of $\mathrm{MoS}_{2}$ using $676.4 \mathrm{~nm}(1.83 \mathrm{eV})$, $647.1 \mathrm{~nm}(1.92 \mathrm{eV})$, and $530.9 \mathrm{~nm}(2.34 \mathrm{eV})$ excitation lasers whose energy is either near or just out of the edge of the $\mathrm{MoS}_{2}$ absorption region [86]. Typically resonance Raman Scattering is excited by use of a He-Ne $632.8 \mathrm{~nm}(1.96 \mathrm{eV})$ laser whose energy is between two absorption bands $\mathrm{A}(\sim 1.9 \mathrm{eV})$ and $\mathrm{B}$ $(\sim 2.1 \mathrm{eV})$, and falls in the region of electronic transition. The resonance Raman spectra show that multiple modes are shifted from the first-order Raman frequencies, which suggests that the electronic transitions are strongly coupled with phonon modes. There are several reports of this resonant Raman scattering phenomenon in $\mathrm{MoS}_{2}[78,79,86,91,93,98-100]$. All of these studies are in general agreement on the attribution of the peaks observed in the resonant Raman scattering spectrum, which suggests coupling between vibrational modes and longitudinal acoustic phonon modes.

Table 4. The phonon frequency of Raman-active and LA modes at different points of the Brillouin zone.

\begin{tabular}{ccccc}
\hline & \multicolumn{4}{c}{ Phonon Frequency $\left(\mathrm{cm}^{-1}\right)$} \\
\cline { 2 - 5 } $\mathrm{A}_{1 \mathrm{~g}}$ & $\mathbf{\Gamma}[\mathbf{8 0 , 8 5 , 8 6 ]}$ & $\mathbf{M}[\mathbf{9 7}]$ & $\mathbf{M}[\mathbf{8 4}]$ & $\mathbf{K}[\mathbf{8 4}]$ \\
\cline { 2 - 5 } $\mathrm{E}_{2 \mathrm{~g}}{ }^{1}$ & 409 & 397 & 412 & 408 \\
$\mathrm{E}_{1 \mathrm{~g}}$ & 383 & 373 & 370 & 345 \\
& 287 & 294 & 330 & 336 \\
$\mathrm{E}_{2 \mathrm{~g}}{ }^{2}$ & 34 & & 309 & $241(\mathrm{LA})$ \\
LA & & - & $233(\mathrm{LA})$ & $192(\mathrm{ZA})$ \\
\hline
\end{tabular}

The resonant Raman spectra are mainly due to contributions from the second order Raman processes at the high symmetry $\Gamma, M$, and $\mathrm{K}$ points of the Brillouin zone. Inelastic neutron scattering data have shown that the energy dispersion of the $\mathrm{E}_{1 \mathrm{~g}}, \mathrm{E}_{2 \mathrm{~g}}{ }^{1}$, and $\mathrm{A}_{1 \mathrm{~g}}$ modes in the $\Gamma-\mathrm{M}$ (from center to the [100] plane (shown in Figure 4b)) direction is relatively small [97]. From the measurement of resonance Raman spectra in Table 4, the line of the $A_{1 g}(M)$ mode almost corresponds to the calculated value of $A_{1 g}$ 
(Г) $\left(409 \mathrm{~cm}^{-1}\right)$ mode. Similarly, the estimated value of the $\mathrm{E}_{2 \mathrm{~g}}{ }^{1}(\mathrm{M})$ mode is a little smaller than the calculated value of the $\mathrm{E}_{2 \mathrm{~g}}{ }^{1}(\Gamma)\left(383 \mathrm{~cm}^{-1}\right)$ mode, which is consistent with Coulombic force theory [38]. However, as shown in Table 4, the energy of the $\mathrm{E}_{2 \mathrm{~g}}{ }^{2}$ rigid layer mode at the $\mathrm{M}$ points increases considerably in the $\Gamma-\mathrm{M}$ direction due to the aforementioned longitudinal acoustic mode LA (M) interaction [97]. The phonon dispersion has three acoustic modes, whose frequencies linearly depend on the wave-vector. They play an important role in the resonance Raman spectrum of $\mathrm{MoS}_{2}$. The longitudinal acoustic (LA), and transverse acoustic (TA) modes are due to in-plane vibration. They both have a linear dispersion and higher energy than the out-of-plane acoustic (ZA) mode [38]. The dispersion of the LA, TA, and ZA modes flatten at the border of the Brillouin zone near the high-symmetry $\mathrm{M}$ point. Even though it is hard to observe in bulk $\mathrm{MoS}_{2}$ as shown in Figure 8, this LA (M) mode has been observed in $\mathrm{MoS}_{2}$ nanoparticles [79], $\mathrm{MoS}_{2}$ monolayer [101], few layers [93], and their aqueous suspension [21]. Hence the peak at $\sim 230 \mathrm{~cm}^{-1}$ is related to the structural defect-induced scattering in $\mathrm{MoS}_{2}$ [93]. Lack of this feature may confirm relatively good crystal quality for the natural $\mathrm{MoS}_{2}$ sample [102].

The profile of many of the first- and second-order peaks between $100 \mathrm{~cm}^{-1}$ and $700 \mathrm{~cm}^{-1}$ can be seen in Figure 8. One of the most pronounced peaks is the multi-modeband at $466 \mathrm{~cm}^{-1}$. Generally it is attributed to the $2 \mathrm{LA}(\mathrm{M})$ mode at $\sim 450 \mathrm{~cm}^{-1}[78,79,86]$, and the normally IR-active $\mathrm{A}_{2 \mathrm{u}}(\Gamma)$ phonon mode near $466 \mathrm{~cm}^{-1}$ [97]. In addition, K. Golasa et al. proposed a new phonon mode XA around $180 \mathrm{~cm}^{-1}$ [100]. They suggested that the high energy component of the $466 \mathrm{~cm}^{-1}$ peak is rather due to a combined mode $\mathrm{E}_{1 \mathrm{~g}}$ $(\mathrm{M})+\mathrm{XA}(\mathrm{M})$, even if the $\mathrm{E}_{1 \mathrm{~g}}$ is forbidden by back scattering geometry. Similarly, the $\mathrm{E}_{1 \mathrm{~g}}+\mathrm{LA}(\mathrm{M})$ mode at $528 \mathrm{~cm}^{-1}$ has been observed in many different samples. Contrary to previous studies [79,86,91,93,101,103], which simply assign the peak at $\sim 645 \mathrm{~cm}^{-1}$ as $\mathrm{A}_{1 \mathrm{~g}}(\mathrm{M})+\mathrm{LA}(\mathrm{M})$, K. Golasa et al. [100,104] suggest it is composed of two components: the high-wavenumber part is due to the $E_{1 g}+2 X A(M)$ modes and the low-wavenumber one, which is marked as " $\mathrm{S}$ " in Figure 8, is the real $\mathrm{A}_{1 \mathrm{~g}}(\mathrm{M})+\mathrm{LA}(\mathrm{M})$ process. In this way, G. L. Frey's description of the $641 \mathrm{~cm}^{-1}$ peak shift to low-energy in $\mathrm{MoS}_{2}$ nanoparticles can be explained [98].

There are multiple emergent modes around the two first-order Raman active modes $\mathrm{E}_{2 \mathrm{~g}}{ }^{1}$ and $\mathrm{A}_{1 \mathrm{~g}}$ in Figure 8. Three additional bands marked "a," "b," and "c" are in the vicinity of the $\mathrm{E}_{2 \mathrm{~g}}{ }^{1}$ and $\mathrm{A}_{1 \mathrm{~g}}$ peaks $[78,93,98,100,104]$. The "a" band is at $\sim 395 \mathrm{~cm}^{-1}$, which is not shown in Figure 8 but has been observed by Sekine et al., Livneh et al., and Golasa et al. [78,98,104]. The mode labeled "b" at $420 \mathrm{~cm}^{-1}$ is attributed to a two-phonon Raman scattering process $\omega_{b}=\omega_{Q A}+\omega_{T O}$. It involves the successive emission of a dispersive longitudinal quasi-acoustic (QA) phonon and a dispersionless transverse optical (TO) phonon. The QA phonon belongs to the $\Delta_{2}$ branch along the $\mathrm{c}$ axis (ending in the $\mathrm{B}_{2 \mathrm{~g}}{ }^{2}$ inactive mode at the $\Gamma$ point). The TO phonon has a finite vector near the $\Gamma$ point (with $\mathrm{E}_{2 \mathrm{u}}{ }^{1}$ symmetry) of the $\Delta_{6}$ branch along the $\mathrm{c}$ axis [97]. Using this, Livneh explains the $\sim 5 \mathrm{~cm}^{-1}$ difference in the " $\mathrm{b}$ " mode between the Stokes and anti-Stokes Raman spectra [98]. Therefore, the band denoted as "c" at $\sim 377 \mathrm{~cm}^{-1}$ corresponds to this TO phonon. However, unlike the IR spectrum result [80], the "c" band was directly considered to be the $\mathrm{E}_{1}{ }^{2}$ mode in Sekine's measurement [78]. P. N. Ghosh et al.'s calculation based on the vdW interlayer model also obtained $\mathrm{E}_{1} \mathrm{u}^{2}$ at $2 \mathrm{~cm}^{-1}$ lower than the $\mathrm{E}_{2 \mathrm{~g}}{ }^{1}$ mode [105], but they suggest the probability of long-range Coulombic interaction. Finally, A. M. Sanchez et al. made ab initio 
calculations considering long-range Coulombic interaction, which showed that the $\mathrm{E}_{1 \mathrm{u}}{ }^{2}$ mode is $3 \mathrm{~cm}^{-1}$ higher than $\mathrm{E}_{2 \mathrm{~g}}{ }^{1}$ mode [38]. The IR-active mode $\mathrm{E}_{1 \mathrm{u}^{2}}$ can be experimentally observed at the high wavenumber side of the $\mathrm{E}_{2 \mathrm{~g}}{ }^{1}$ mode from the resonant Raman scattering in Livneh's work [98]. Another Raman-inactive mode, $\mathrm{B}_{1 \mathrm{u}}$, which is the Davydov pair of $\mathrm{A}_{1 \mathrm{~g}}$ modes, is also observed at the low-wavenumber side of the Aig band [78,93,98].

Resonance Raman spectra taken on different $\mathrm{MoS}_{2}$ thicknesses from monolayer to bulk are shown in Figure 8. One of the most significant differences is that almost all the resonance peaks are broadened and the relative intensity change as a result of thickness decreases, as was previously reported [91]. The change in the shape and location of the resonance modes at $\sim 465 \mathrm{~cm}^{-1}$ and

$643 \mathrm{~cm}^{-1}$ have been explained in detail by K. Golasa et al. [100,104]. In addition, the resonance Raman modes higher than $500 \mathrm{~cm}^{-1}$ are hardly observed in the spectrum of monolayer MoS2. There are several possible reasons for this result. The most reasonable possibility is that the long-range acoustic modes in monolayer $\mathrm{MoS}_{2}$ are strongly affected by interactions with the substrate [99,104]. In the bulk case, only a relatively tiny amount of atoms in the whole sample directly interact with the substrate, and the substrate therefore has little impact on the lattice vibrations. Under resonance conditions, the modification of acoustic modes by the substrate causes the suppression of most multi-phonon processes.

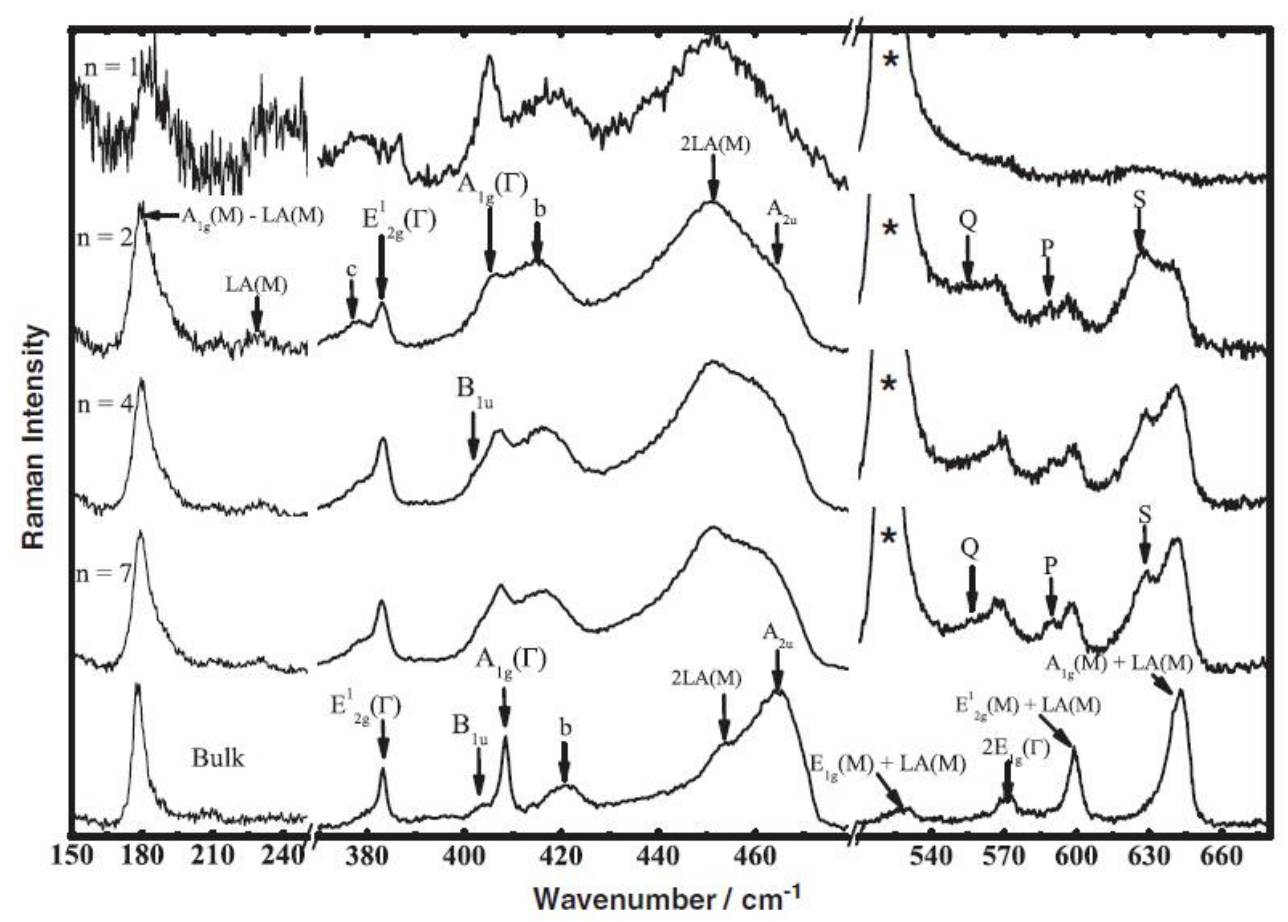

Figure 8. Resonant Raman spectra of different thickness $\mathrm{MoS}_{2}$ using $632.8 \mathrm{~nm}$ laser in room temperature [93]. Reprinted figure with permission from [Chakraborty, B.; Matte, H.S.S.R.; Sood, A.K.; Rao, C.N.R. Layer-dependent resonant Raman scattering of a few layer $\mathrm{MoS}_{2}$. J. Raman Spectrosc. 2013, 44, 92-96.] Copyright (2013) by John Wiley and Sons.

Another result of the resonant spectra is that mode " $\mathrm{b}$ " at $\sim 420 \mathrm{~cm}^{-1}$ seems to still be present in monolayer $\mathrm{MoS}_{2}$ at room temperature $[93,101,104]$. However, there is no quasi-acoustical mode 
present in the monolayer limit, thus the behavior contradicts its assignment to the combined process involving the quasi-acoustic phonons. Therefore, mode " $b$ " should not be present in the monolayer $\mathrm{MoS}_{2}$ spectrum at all. Based on the data from K. Golasa, the peak " $\mathrm{b}$ " at $\sim 420 \mathrm{~cm}^{-1}$ clearly disappears from the spectrum in the monolayer $\mathrm{MoS}_{2}$ at $4.2 \mathrm{~K}$. This observation also seems to suggest that a new model is needed to explain the apparent presence of the mode "b" at $\sim 420 \mathrm{~cm}^{-1}$.

Finally, the intensity of resonance Raman modes have an observed temperature dependence in some studies [85,98-100,106]. For example, J. M. Chen and C. S. Wang had detected both the dependence of the second-order Raman modes at room temperature $(300 \mathrm{~K})$ and liquid nitrogen temperature $(80 \mathrm{~K})$. These resonance modes can be explained based on the following relations:

Difference process: $I \propto n(1, T) \times[n(2, T)+1]$

Combination process: $I \propto[n(1, T)+1] \times[n(2, T)+1]$, where $\mathrm{n}(\mathrm{i}, \mathrm{T})=\frac{1}{\mathrm{e}^{\mathrm{h} \omega_{\mathrm{i}} / \mathrm{kT}}-1}$ is the phonon number related to temperature $\mathrm{T}$ for phonons with frequency $\omega_{\mathrm{i}}[85]$. Based on these absorption and excitation processes for phonons, they tentatively identify two low frequency peaks at $150 \mathrm{~cm}^{-1}$ and $188 \mathrm{~cm}^{-1}$ and three high frequency peaks at $567 \mathrm{~cm}^{-1}, 750 \mathrm{~cm}^{-1}$, and $816 \mathrm{~cm}^{-1}$.

\section{Conclusions}

The emergence of 2D materials has led to increased attention on correlating the structural, optical, and optoelectronic properties of thin $\mathrm{MoS}_{2}$ layers. Although these properties have been studied for bulk $\mathrm{MoS}_{2}$ since the $1960 \mathrm{~s}$, the discovery of graphene has led to more interest in the investigation of these properties for 2D thin films of $\mathrm{MoS}_{2}$. Since the discovery of the relationship between Raman and photoluminescence (PL) spectra with the film thickness of $\mathrm{MoS}_{2}$ in 2010, these optical spectra have become reliable and popular signals for the identification of mono- or few-layered $\mathrm{MoS}_{2}$ structures.

\section{Acknowledgements}

Yoke Khin Yap acknowledges the support from the National Science Foundation (Award number DMR-1261910).

\section{Conflicts of Interest}

The authors declare no conflict of interest.

\section{References}

1. Novoselov, K.S.; Geim, A.K.; Morozov, S.V.; Jiang, D.; Zhang, Y.; Dubonos, S.V.; Grigorieva1, I.V.; Firsov, A.A. Electric field effect in atomically thin carbon films. Science 2004, 306, 666-669.

2. Novoselov, K.S.; Jiang, D.; Schedin, F.; Booth, T.J.; Khotkevich, V.V.; Morozov, S.V.; Geim, A.K. Two-dimensional atomic crystals. PNAS 2005, 102, 10451-10453.

3. Geim, A.K.; Grigorieva, I.V. Van der Waals heterostructures. Nature 2013, 499, 419-425.

4. Castro Neto, A.H.; Guinea, F.; Peres, N.M.R.; Novoselov, K.S.; Geim, A.K. The electronic properties of graphene. Rev. Mod. Phys. 2009, 81, 109-162. 
5. Elias, D.C.; Gorbachev, R.V.; Mayorov, A.S.; Morozov, S.V.; Zhukov, A.A.; Blake, P.; Ponomarenko, L.A.; Grigorieva, I.V.; Novoselov, K.S.; Guinea, F. et al. Dirac cones reshaped by interaction effects in suspended graphene. Nat. Phys. 2011, 7, 701-704.

6. Chen, H.; Müller, M.B.; Gilmore, K.J.; Wallace, G.G.; Li, D. Mechanically Strong, Electrically Conductive, and Biocompatible Graphene Paper. Adv. Mater. 2008, 20, 3557-3561.

7. Lin, M.W.; Ling, C.; Zhang, Y.; Yoon, H.J.; Cheng, M.M.-C.; Agapito, L.A.; Kioussis, N.; Widjaja, N.; Zhou, Z. Room-temperature high on/off ratio in suspended graphene nanoribbon field-effect transistors. Nanotechnology 2011, 22, 265201.

8. Song, L.; Ci, L.J.; Lu, H.; Sorokin, P.B.; Jin, C.; Ni, J.; Kvashnin, A.G.; Kvashnin, D.G.; Lou, J.; Yakobson, B.I.; Ajayan, P.M. Large Scale Growth and Characterization of Atomic Hexagonal Boron Nitride Layers. Nano Lett. 2010, 10, 3209-3215.

9. Kim, K.K.; Hsu, A.; Jia, X.; Kim, S.M.; Shi, Y.; Hofmann, M.; Nezich, D.; Rodriguez-Nieva, J.F.; Dresselhaus, M.; et al. Synthesis of Monolayer Hexagonal Boron Nitride on $\mathrm{Cu}$ Foil Using Chemical Vapor Deposition. Nano Lett. 2012, 12, 161-166.

10. Lee, C.H.; Qin, S.Y.; Savaikar, M.A.; Wang, J.; Hao, B.; Zhang, D.; Banyai, D.; Jaszczak, J.A.; Clark, K.W.; et al. Room-Temperature Tunneling Behavior of Boron Nitride Nanotubes Functionalized with Gold Quantum Dots. Adv. Mater. 2013, 25, 4544-4548.

11. Lee, C.H.; Xie, M.; Kayastha, V.; Wang, J.; Yap, Y.K. Patterned Growth of Boron Nitride Nanotubes by Catalytic Chemical Vapor Deposition. Chem. Mater. 2010, 22, 1782-1787.

12. Wang, J.S.; Lee, C.H.; Yap, Y.K. Recent advancements in boron nitride nanotubes. Nanoscale 2010, 2, 2028-2034.

13. Wang, J.S.; Kayastha, V.K.; Yap, Y.K.; Fan, Z.; Lu, J.G.; Pan, Z.; Ivanov, I.N.; Puretzky, A.A.; Geohegan, D.B. Low temperature growth of boron nitride nanotubes on substrates. Nano Lett. 2005, 5, 2528-2532.

14. Wilson, J.A.; Yoffe, A.D. The transition metal dichalcogenides discussion and interpretation of the observed optical, electrical and structural properties. Adv. Phys. 1969, 18, 193-335.

15. Yoffe, A.D. Layer compounds. Annu. Rev. Mater. Sci. 1973, 3, 147-170.

16. Radisavljevic, B.; Radenovic, A.; Brivio, J.; Giacometti, V.; Kis, A. Single-layer MoS 2 transistors. Nat. Nanotech. 2011, 6, 147-150.

17. Yoon, Y.; Ganapathi, K.; Salahuddin, S. How good can monolayer $\mathrm{MoS}_{2}$ transistors be? Nano Lett. 2011, 11, 3768-3773.

18. Frindt, R.F.; Yoffe, A.D. Physical Properties of Layer Structures: Optical Properties and Photoconductivity of Thin Crystals of Molybdenum Disulphide. Proceedings A 1963, 27369-27383.

19. Dickinson, R.G. Pauling, L. The crystal structure of molybdenite. J. Am. Chem. Soc. 1923, 45, 1466-1471.

20. Jellinek, P.; Brauer, G.; Müller, H. Molybdenum and Niobium Sulphides. Nature 1960, 185, 185376-185377.

21. Jiménez Sandoval, S.; Yang, D.; Frindt, R.F.; Irwin, J.C. Raman study and lattice dynamics of single molecular layers of MoS2. Phys. Rev. B 1991, 44, 3955-3962. 
22. Wypych, F; Schollhorn, R. 1T-Mos2, a New Metallic Modification of Molybdenum-Disulfide. $J$. Chem. Soc. -Chem. Commun. 1992, 19, 1386-1388.

23. Enyashin, A.N.; Yadgarov, L.; Houben, L.; Popov, I.; Weidenbach, M.; Tenne, R.; Bar-Sadan, M.; Seifert, G. New Route for Stabilization of 1T-WS 2 and $\mathrm{MoS}_{2}$ Phases. J. Phys. Chem. C 2011, 115, 24586-24591.

24. Eda, G.; Yamaguchi, H.; Voiry, D.; Fujita, T.; Chen, M.; Chhowalla, M. Photoluminescence from chemically exfoliated MoS 2 . Nano Lett. 2011, 11, 5111-5116.

25. Mattheiss, L.F. Band Structures of Transition-Metal-Dichalcogenide Layer Compounds. Phys. Rev. B 1973, 8, 3719-3740.

26. Shepherd, F.R.; Williams, P.M. Photoemission studies of the band structures of transition metal dichalcogenides. I. Groups IVA and IVB. J. Phys. C: Solid State Phys. 1974, 4416, 74416-74426.

27. Shepherd, F.R.; Williams, P.M. Photoemission studies of the band structures of transition metal dichalcogenides. II. Groups VA and VIA. J. Phys. C: Solid State Phys. 1974, 4427, 74427-74439.

28. Bromley, R.A.; Murray, R.B.; Yoffe, A.D. The band structures of some transition metal dichalcogenides. III. Group VIA trigonal prism materials. J. Phys. C: Solid State Phys. 1972, doi:10.1088/0022-3719/5/7/007.

29. Bromley, R.A. A Semi-Emprical Tight-Banding Calculation of the Band Structure of MoS 2 . Phys. Lett. 1970, 33, 33242-33243.

30. Mattheiss, L.F. Energy Bands for $2 \mathrm{H}-\mathrm{NbSe}_{2}$ and $2 \mathrm{H}-\mathrm{MoS}_{2}$. Phys. Rev. Lett. 1973, 30, 784-787.

31. Edmondson, D.R. Electronic band structure of the layer-type crystal $2 \mathrm{H}-\mathrm{MoS}_{2}$. Solid State Commun. 1972, 10, 101085-101088.

32. Coehoorn, R.; Haas, C.; de Groot, R.A. Electronic structure of $\mathrm{MoSe}_{2}, \mathrm{MoS}_{2}$, and WSe2. II. The nature of the optical band gaps. Phys. Rev. B 1987, 35, 6203-6206.

33. Reshak, A.H.; Auluck, S. Band structure and optical response of $2 \mathrm{H}-\mathrm{MoX} 2$ compounds $(\mathrm{X}=\mathrm{S}, \mathrm{Se}$, and Te). Phys. Rev. B 2005, doi:10.1103/PhysRevB.71.155114.

34. Li, T.S.; Galli, G.L. Electronic properties of $\mathrm{MoS}_{2}$ nanoparticles. J. Phys. Chem. C 2007, 111, 16192-16196.

35. Lebègue, S.; Eriksson, O. Electronic structure of two-dimensional crystals from ab initio theory. Phys. Rev. B 2009, 79, 115409.

36. Kuc, A.; Zibouche, N.; Heine, T. Influence of quantum confinement on the electronic structure of the transition metal sulfide TS2. Phys. Rev. B 2011, 83, 245213.

37. Ding, Y.; Wang, Y.; Ni, J.; Shi, L.; Shi, S.; Tang, W. First principles study of structural, vibrational and electronic properties of graphene-like MX2 $(\mathrm{M}=\mathrm{Mo}, \mathrm{Nb}, \mathrm{W}, \mathrm{Ta} ; \mathrm{X}=\mathrm{S}, \mathrm{Se}, \mathrm{Te})$ monolayers. Phys. B: Condens. Matt. 2011, 406, 2254-2260.

38. Molina-Sánchez, A.; Wirtz, L. Phonons in single-layer and few-layer $\mathrm{MoS}_{2}$ and $\mathrm{WS}_{2}$. Phys. Rev. B 2011, 84, 155413.

39. Kadantsev, E.S.; Hawrylak, P. Electronic structure of a single $\mathrm{MoS}_{2}$ monolayer. Solid State Commun. 2012, 152, 909-913. 
40. Yun, W.S.; Han, S.W.; Hong, S.C.; Kim, I.G.; Lee. J.D. Thickness and strain effects on electronic structures of transition metal dichalcogenides: $2 \mathrm{H}_{-} \mathrm{MX}_{2}$ semiconductors $(\mathrm{M}=\mathrm{Mo}, \mathrm{W} ; \mathrm{X}=\mathrm{S}$, Se, Te). Phys. Rev. B 2012, doi:10.1103/PhysRevB.85.033305.

41. Kam, K.K.; Parklnclon, B.A. Detailed Photocurrent Spectroscopy of the Semiconducting Grouping VI Transition Metal Dichalcogenides. J. Phys. Chem. 1982, 86, 86463-86467.

42. Coehoorn, R.; Haas, C.; Dijkstra, J.; Flipse, C.J.; de Groot, R.A.; Wold, A. Electronic structure of $\mathrm{MoSe}_{2}, \mathrm{MoS}_{2}$, and WSe2. I. Band-structure calculations and photoelectron spectroscopy. Phys. Rev. B 1987, 35, 6195-6202.

43. Kobayashi, K.; Yamauchi, J. Electronic structure and scanning-tunneling-microscopy image of molybdenum dichalcogenide surfaces. Phys. Rev. B Condens. Matt. 1995, 51, 17085-17095.

44. Frey, G.L.; Elani, S.; Homyonfer, M.; Feldman, Y.; Tenne, R. Optical-absorption spectra of inorganic fullerenelike $\mathrm{MS}_{2}(\mathrm{M}=\mathrm{Mo}, \mathrm{W})$. Phys. Rev. B 1998, doi:10.1103/PhysRevB.57.6666.

45. Böker, T.; Severin, R.; Müller, A.; Janowitz, C.; Manzke, R.; Voß, D.; Krüger, P.; Mazur, A.; Pollmann, J. Band structure of $\mathrm{MoS}_{2}, \mathrm{MoSe}_{2}$, and $\alpha-\mathrm{MoTe}_{2}$ : Angle-resolved photoelectron spectroscopy and ab initio calculations. Phys. Rev. B 2001, doi:10.1103/PhysRevB.64.235305.

46. Mak, K.F.; Lee, C.; James, H.; Jie, S.; Heinz, T.F. Atomically Thin MoS2: A New Direct-Gap Semiconductor. Phys. Rev. Lett. 2010, doi:10.1103/PhysRevLett.105.136805.

47. Splendiani, A.; Sun, L.; Zhang, Y.; Li, T.; Kim, J.; Chim, C.-Y.; Galli, G.; Wang, F. Emerging photoluminescence in monolayer MoS2. Nano Lett. 2010, 10, 1271-1275.

48. Jin, W.; Yeh, P.-C.; Zaki, N.; Zhang, D.; Sadowski, J.T.; Al-Mahboob, A.; van der Zande, A.M. Chenet, D.A.; Dadap, J.I.; Herman, I.P. Direct Measurement of the Thickness-Dependent Electronic Band Structure of $\mathrm{MoS}_{2}$ Using Angle-Resolved Photoemmision Spectroscopy. Phys. Rev. Lett. 2013, 111, 106801.

49. Sundaram, R.S.; Engel, M.; Lombardo, A.; Krupke, R.; Ferrari, A.C.; Avouris, Ph.; Steiner, M. Electroluminescence in single layer MoS2. Nano Lett. 2013, 13, 1416-1421.

50. Eknapakul, T.; King, P.D.; Lombardo, A.; Krupke, R.; Ferrari, A.C.; Avouris, P.; Steiner, M. Electronic structure of a quasi-freestanding $\mathrm{MoS}_{2}$ monolayer. Nano Lett. 2014, 14, 1312-1316.

51. Sandormirskii, V.B. Dependence of the forbiffen-band width of semiconducting films on their thickness and temperature. SOVIET PHYS. JETP 1963, 16, 1630-1631.

52. Sandomirskii, V.B. Quantum Size Effect in a Semimetal Film. SOVIET PHYS. JETP 1967, 25, 101-106.

53. Consadori, F.; Frindt, R.F. Crystal Size Effects on the Exciton Absorption Spectrum of WSe2. Phys. Rev. B 1970, 2, 4893-4896.

54. Evans, B.L.; Young, P.A. Exciton spectra in thin crystals the diamagnetic effect. Proc. Phy. Soc. 1967, 91, 91475-91482.

55. Yoffe, A.D. Low-dimensional systems: Quantum size effects and electronic properties of semiconductor microcrystallites (zero-dimensional systems) and some quasi-two-dimensional systems. Adv. Phys. 1993, 42, 173-262.

56. Williams, P.M.; Shepherd, F.R. He II photoemission studies of transition metal dichalcogenides. J. Phys. C: Solid State Phys. 1973, doi:10.1088/0022-3719/6/1/011. 
57. Setyawan, W.; Curtarolo, S. High-throughput electronic band structure calculations: Challenges and tools. Comput. Mater. Sci. 2010, 49, 299-312.

58. Yin, Z.; Li, H.; Li, H.; Jiang, L.; Shi, Y.; Sun, Y.; Lu, G.; Zhang, Q.; Chen, X.; Zhang, H. Single-layer $\mathrm{MoS}_{2}$ phototransistor. ACS Nano 2012, 6, 674-680.

59. Beal, A.R.; Knights, J.C.; Liang, W.Y. Transmission spectra of some transition metal dichalcogenides. II. Group VIA: Trigonal prismatic coordination. J. Phys. C: Solid State Phys. 1972, 5, 53540-53551.

60. Kasowski, R.V. Band Structure of $\mathrm{MoS}_{2}$ and $\mathrm{NbS}_{2}$. Phys. Rev. Lett. 1973, 30, 1175-1178.

61. Bullett, D.W. Electronic Band-Structure and Bonding in Transition-Metal Layered Dichalcogenides by Atomic Orbital Methods. J. Phys. C-Solid State Phys. 1978, 11, 4501-4514.

62. Tanaka, M.; Fukutani, H.; Kuwabara, G. Excitons in VI B Transition Metal Dichalcogenides. $J$. Phys. Soc. Japan 1978, 45, 1899-1904.

63. Khan, M.R.; Goldsmith, G.J. Optical, Electro-Optical and Transport-Properties of MoS2. II Nuovo Cimento D 1983, 2, 665-686.

64. Wood, K.; Pendry, J.B. Layer Method for Band-Structure of Layer Compounds. Phys. Rev. Lett. 1973, 31, 1400-1403.

65. Hind, S.P.; Lee, P.M. KKR calculations of the energy bands in $\mathrm{NbSe}_{2}, \mathrm{MoS}_{2}$ and alpha $\mathrm{MoTe}_{2} . J$. Phys. C: Solid State Phys. 1980, 13, 13349-13357.

66. Ramasubramaniam, A. Large excitonic effects in monolayers of molybdenum and tungsten dichalcogenides. Phys. Rev. B 2012, 86, 115409.

67. Huisman, R.; De Jonge, R.; Haas, C.; Jellinek, F. Trigonal-Prismatic Coordination in Solid Compounds of Transition Metals. J. Phys. C: Solid State Phys. 1971, 356, 56-66.

68. Jiang, H. Electronic Band Structures of Molybdenum and Tungsten Dichalcogenides by the GW Approach. J. Phys. Chem. C 2012, 116, 7664-7671.

69. Cheiwchanchamnangij, T.; Lambrecht, W.R.L. Quasiparticle band structure calculation of monolayer, bilayer, and bulk MoS 2. Phys. Rev. B 2012, 85, 205302.

70. Komsa, H.-P.; Krasheninnikov, A.V. Effects of confinement and environment on the electronic structure and exciton binding energy of $\mathrm{MoS}_{2}$ from first principles. Phys. Rev. B 2012, 86, 241201.

71. Shi, H.; Pan, H.; Zhang, Y.-W.; Yakobson. B.I. Quasiparticle band structures and optical properties of strained monolayer $\mathrm{MoS}_{2}$ and $\mathrm{WS}_{2}$. Phys. Rev. B 2013, 87, 155304.

72. Qiu, D.Y.; da Jornada, F.H.; Louie, S.G. Optical Spectrum of $\mathrm{MoS}_{2}$ : Many-Body Effects and Diversity of Exciton States. Phys. Rev. Lett. 2013, 111, 216805.

73. Ye, Z.; Cao, T.; O’Brien, K.; Zhu, H.; Yin, X.; Wang, Y.; Louie, S.G.; Zhang, X. Probing excitonic dark states in single-layer tungsten disulphide. Nature 2014, 513, 214-218.

74. Ho, C.H.; Wu, C.S.; Huang, Y.S.; Liao, P.C.; Tiong, K.K. Temperature dependence of energies and broadening parameters of the band-edge excitons of Mo1-x $\mathrm{WxS}_{2}$ single crystals. J. Phys.-Condens. Matt. 1998, 10, 9317-9328.

75. Acrivos, J.V.; Liang, W.Y.; Wilson, J.; Yoffe, A. Optical studies of metal-semiconductor transmutations produced by intercalation. J. Phys. C: Solid State Phys. 1971, 4, L18-L20. 
76. Beal, A.R.; Hughes, H.P. Kramers-Kronig Analysis of the Reflectivity Spectra of 2h-MoS2, 2h-MoSe2 and 2h-MoTe2. J. Phys. C-Solid State Phys. 1979, 12, 881-890.

77. Fortin, E.; Raga, F. Excitons in Molybdenum-Disulfide. Phys. Rev. B 1975, 11, 905-912.

78. Sekine, T.; Uchinokura, K.; Nakashizu, T.; Matsuura, E.; Yoshizaki. R. Dispersive Raman Mode of Layered Compound 2H MoS 2 under the resonant condition. J. Phys. Soc. Japan 1984, 53, 811-818.

79. Frey, G.L.; Tenne, R.; Matthews, M.J.; Dresselhaus, M.S.; Dresselhaus, G. Raman and resonance Raman investigation of $\mathrm{MoS}_{2}$ nanoparticles. Phys. Rev. B 1999, 60, 2883-2892.

80. Verble, J.L.; Wieting, T.J. Lattice Mode Degeneracy in $\mathrm{MoS}_{2}$ and Other Layer Compounds. Phys. Rev. Lett. 1970, 25, 362-365.

81. Wieting, T.J.; Verble, J.L. Infrared and Raman Studies of Long-Wavelength Optical Phonons in Hexagonal MoS2. Phys. Rev. B 1971, 3, 4286-4292.

82. Lee, C.; Yan, H.; Brus, L.E.; Heinz, T.F.; Hone, J.; Ryu, S. Anomalous lattice vibrations of singleand few-layer MoS 2 . ACS Nano 2010, 4, 2695-2700.

83. Agnihotri, O.P.; Sehgal, H.K.; Garg, A.K. Laser Excited Raman Spectra of Gr. VI Semiconducting Compounds.pdf. Solid state Commun. 1973, 12, 12135-12138.

84. Tsachi, L.; Spanier, J.E. A Comprehensive Spectral Analysis of Multiphonon Resonant Raman Scattering in $2 \mathrm{H}-\mathrm{MoS}_{2}$. arXiv 2014.

85. Chen, J.M.; Wang, C.S. Second order Raman spectrum of MoS2. Solid State Commun. 1974, 14, 14857-14860.

86. Stacy, A.M.; Hodul, D.T. Raman spectra of IVB and VIB transition metal disulfides using laser energies near the absorption. J. Phys. Chem. Solids 1985, 46, 405-409.

87. Scherer, J.R.; Bailey, G.F.; Kint, S. Simple Optical Geometry for Obtaining Raman Back Scattering. Analy. Chem. 1971, 43, 1917-1918.

88. Shriver, D.F.; Dunn, J.B.R. The Backscattering Geometry for Raman Spectroscopy of Colored Materials. Appl. Spectrosc. 1974, 28, 319-323.

89. Verble, J.L.; Wietling, T.J. Rigid-layer lattice vibrations and van der waals bonding in hexagonal MoS. Solid State Commun. 1972, 11, 11941-11944.

90. Bromley, R.A. The lattice vibrations of the $\mathrm{MoS}_{2}$ structure. Philosoph. Mag. 1971, 23, 1417-1427.

91. Li, H.; Zhang, Q.; Yap, C.C.R.; Tay, B.K.; Edwin, T.H.T.; Olivier, A.; Baillargeat, D. From Bulk to Monolayer $\mathrm{MoS}_{2}$ : Evolution of Raman Scattering. Adv. Function. Mater. 2012, 22, 1385-1390.

92. Bertrand, P. Surface-phonon dispersion of $\mathrm{MoS}_{2}$. Phys. Rev. B 1991, 44, 5745-5749.

93. Chakraborty, B.; Matte, H.S.S.R.; Sood, A.K.; Rao, C.N.R. Layer-dependent resonant Raman scattering of a few layer $\mathrm{MoS}_{2}$. J. Raman Spectrosc. 2013, 44, 92-96.

94. Mrstik, B.; Kaplan, R.; Reinecke, T.L.; Van Hove, M.; Tong, S.Y. Surface-structure determination of the layered compounds $\mathrm{MoS}_{2}$ and $\mathrm{NbSe}_{2}$ by low-energy electron diffraction. Phys. Rev. B 1977, 15, 897-900.

95. Wieting, T.J.; Verble, J.L. Interlayer Bonding and the Lattice Vibrations of $\beta$-GaSe. Phys. Rev. $B$ 1972, 5, 1473-1479. 
96. Gonze, X.; Lee, C. Dynamical matrices, Born effective charges, dielectric permittivity tensors, and interatomic force constants from density-functional perturbation theory. Phys. Rev. B 1996, 55, 10355.

97. Wakabayashi, N.; Smith, H.G.; Nicklow, R.M. Lattice dynamics of hexagonal $\mathrm{MoS}_{2}$ studied by neutron scattering. Phys. Rev. B 1975, 12, 659-663.

98. Livneh, T.; Sterer, E. Resonant Raman scattering at exciton states tuned by pressure and temperature in 2H-MoS2. Phys. Rev. B 2010, 81, 195209.

99. Fan, J.-H.; Gao, P.; Zhang, A.M.; Zhu, B.-R.; Zeng, H.-L.; Cui, X.-D.; He, R.; Zhang, Q.-M. Resonance Raman scattering in bulk $2 \mathrm{H}-\mathrm{MX}_{2}(\mathrm{M}=\mathrm{Mo}, \mathrm{W} ; \mathrm{X}=\mathrm{S}, \mathrm{Se})$ and monolayer $\mathrm{MoS}_{2} . J$. Appl. Phys. 2014, 115, 053527.

100. Gołasa, K.; Grzeszczyk, M.; Leszczyński, P.; Faugeras, C.; Nicolet, A.A.L.; Wysmołek, A.; Potemski, M.; Babiński, A. Multiphonon resonant Raman scattering in MoS2. Appl. Phys. Lett. 2014, 104, 092106.

101. Golasa, K.; Grzeszczyk, M.; Korona, K.P.; Bożek, R.; Binder, J.; Szczytko, J. Wysmołek, A. Babiński, A. Optical Properties of Molybdenum Disulfide (MoS2). ACTA PHYS. POLONICA A 2013, 124, 849-851.

102. McDevitf, N.T.; Zabinski, J.S.; Donley, M.S.; Bultman, J.E. Disorder-Induced Low-Frequency Raman Band Observed in Deposited MoS 2 Films. Appl. Spectrosc. 1994, 48, 733-736.

103. Windom, B.C.; Sawyer, W.G.; Hahn, D.W. A Raman Spectroscopic Study of $\mathrm{MoS}_{2}$ and $\mathrm{MoO}_{3}$ : Applications to Tribological Systems. Tribol. Lett. 2011, 42, 301-310.

104. Gołasa, K.; Grzeszczyk, M.; Bożek, R.; Leszczyński, P.; Wysmołek, A.; Potemski, M.; Babiński, A. Resonant Raman scattering in MoS2-From bulk to monolayer. Solid State Commun. 2014, 197, 19753-19756.

105. Ghosh, P.N.; Maiti, C.R. Interlayer Force and Davydov Splitting in $2 \mathrm{H}-\mathrm{MoS}_{2}$. Phys. Rev. B 1983, 28, 2237-2239.

106. Najmaei, S.; Liu, Z.; Ajayan, P.M.; Lou, J. Thermal effects on the characteristic Raman spectrum of molybdenum disulfide $\left(\mathrm{MoS}_{2}\right)$ of varying thicknesses. Appl. Phys. Lett. 2012, 100, 013106.

(C) 2015 by the authors; licensee MDPI, Basel, Switzerland. This article is an open access article distributed under the terms and conditions of the Creative Commons Attribution license (http://creativecommons.org/licenses/by/4.0/). 